\title{
Influence of General Anesthesia on Impulsivity and Learning Ability-Experimental Study
}

\author{
Sircuța Carmen*, Veres Mihaly, Azamfirei Leonard \\ University of Medicine and Pharmacy Tirgu Mures, Romania
}

Objective: To investigate the effect of anesthesia on rats' ability of learning and over their impulsivity.

Material and Methods: We studied eight Wistar adult male rats, test and drug naive subjects. Animals were separated in two groups, group A and B with four members each. Group A included the anesthetized animals. The combination of ketamine, xylazine and piplophen in $2 \mathrm{ml} /$ $\mathrm{kg}$ body weight dosage was used and testing was done 24 hours after anesthesia. Group B was taken as control. The study was conducted using the "Delay discounting" apparatus. Experiments assessing impulsive behavior were conducted using automated operant chambers, equipped with two nose-poke holes (holes where pellets of food were released). Rat's answer was considered touching the nose-poke hole. One answer was rewarded with pellets of food of $45 \mathrm{mg}$ each (small reward), while another hole released five pellets of $45 \mathrm{mg}$ each (high reward). Both types of rewards were presented immediately after rat's answer and were followed for a period of 25 seconds timeout. During the training phase, rats were placed in operant chambers 30 minutes per day, 5 consecutive days. The growing percent of preference for greater reward indicates learning.For the testing phase the procedure was similar, but a delay was introduced before the release of the big reward. During this phase, the preference for higher reward was indicative for non-impulsive behaviour.

Results: The results didn't show significant statistically differences between the two groups.

Conclusions: Anesthesia had no effect on learning ability nor on impulsivity.

Keywords: learning ability, impulsivity, rat, anesthesia

Received: 04 August 2016 / Accepted: 15 September 2016

\section{Introduction}

The main purpose of the general anesthesia is to facilitate the surgical procedure. This is achieved with the help of anesthetic drugs, which all have a common feature-they all modulate neuronal activity, although they present a great diversity and biochemical heterogenity. This substances depress the central nervous system enough to alow invazive, unpleasent and painful procedures to take place [1].

However general anesthesia is not a procedure without consequences; mainly incriminated adverse manifestation after an anesthetic procedure being behavioural changes and postoperative cognitive disfunction. This two entities can also affect young and old patients. Especially postoperative cognitive disfunction was described as adverse cerebral effects after surgery and anesthesia that affect old people [2].

Behavioural changes after a surgical-anesthestic procedure may be due to new cognitive deficits that appear postoperatively, like memory impairment, ability to solve or combine different types of tasks, impairment of psychomotor dexterity and which compose the postoperative cognitive disfunction (POCD). These may last up to 6 months after the surgery and although it arises immediately after the procedure it must be distinguish by other entities with nervous system involvement like: postoperative delirium or dementia [3]. It seems that incidence of this issue is quite high, varying between $12 \%$ and $25,8 \%$ in people above 60 years old [3-5].

* Correspondence to: Carmen Sircuta

E-mail: carmensircuta@yahoo.com
Still, these cognitive alterations aren't the only changes that occur after a general anesthetic procedure. Especially in pediatric practice, postoperative psychological and behavioural changes like impulsivity, aggresivity, separation anxiety and sleep disorders occur up to $50 \%-60 \%$ of the cases $[6,7]$. It has been studied and shown that these strongly correlates with the degree of preoperative anxiety that the child feels [8]. Impulsivity is one issue that has been studied in adult patient but more intensively in pediatric patient suffering by conditions that include this manifestation and even more intensively in animal studies. It may be described as aberrant social behaviour and maladaptative decision making [9].

As anesthesia evolved and nowadays the combined and multimodal balanced anesthetic procedures which use a combination of clases of anesthetic drugs, concerns that these could increase postoperative impulsivity arised, with most incriminated drugs being benzodiazepine class. It is know that low doses, including therapeutically doses of diazepam do not increase impulsive behaviour; still the effects of higher dose has to be determined [10].

Which is the exact mechanism that leads to neurocognitive malfunction? We have to know that there isn't just one mechanism but there are multiple ways an anesthetic drug can interfere with cognitive processes and even affect them even after postprocedure. It is belived that one of them is the neuronal cell death, which leads to long-term cognitive impairment. Hipercarbia resulted during isoflurane exposure seems to achieve important levels. If the $\mathrm{CO} 2$ levels are high enough and sustained for four hours, neuronal 
death occurs [11]. Another way by which isoflurane could exert it's effects in the POCD occurrence is by impairing neurogenesis in the dentate gyrus in the developing brain. This is an important area for proper functioning of the processes involved in memory and learning [11].

Not only inhalator agents have effects on neurogenesis. Another commonly used anesthetic drug, especially in veterinary practice, ketamine has been reported to cause neurotoxicity by an apoptosis mechanism on neonatal brain. Neonatal exposure to ketamine significantly inhibited the proliferation of neuronal stem cells and astrocytes differentiation but increased neuronal differentiation. The ketamine usage also stopped neuronal migration and granulocyte and astrocytes cell layer growth. These tissue structural changes have translated into a deficit in solving tasks which involved spatial memory, itself dependent of cell integrity in hippocampal regions [12].

While the above mentioned mechanism are responsible for changes met in the neurocognitive field, it is belived that impulsive behaviour is related to frontostriatal circuit which involves the following two brain structures: nucleus accumbens and the prefrontal cortex [13].

Due to the fact that the brain developmental stage of 7 days postnatal age of mice and rats has been considered equivalent to the human brain at 32-36 weeks of gestation, many studies of anesthesia and it's impact are conducted on animals, since there is needed for investigate on brain slices preparations the celluar and molecular changes and process that occur during surgical-anesthetic procedures $[14,15]$.

Still it has to be taken into accout that animal studies have their limitation in human usability like inaccurate comparing of animal studies with human anesthesia practice, or the exact maturational state of the bain in the experimental designs there are such animal studies that suggest that general anesthesia itself may contribute to persistent impairment of learning and memory processes and increase impulsivity [16-17]

When the effect of anesthetics were studied on rodents, it had been shown that combined isoflurane-nitrous oxide anesthesia decreased animals' performance when completing a certain type of test, for example a spatila memory test. The time of completing the maze was prolonged with more incorrect responses than before anesthesia [18].

\section{Aim of the study}

To study the effect of anesthesia on rats' ability of learning and over their impulsive behaviour.

\section{Material and method}

We studied a group of eight Wistar adult male rats weighting between 250-300 grams, test and drug naive subjects (this means that they have never been anesthetized nor completed before none of the following tests, in their life). These were purchased from Charles River Laboratories (Hungary).
All rats were housed in groups of five or six per cage, at $22 \pm 2{ }^{\circ} \mathrm{C}$ under 12 hour day-night schedule (light on at 08:00). Tap water was given ad libitum. The food in lab was freely available until the day before the test; when food was limited to six pellets of food per day (about 20grams) to stimulate rats to conscientious explore the environment, along the delay phase of the experiments. Food release was provided immediately after finishing the daily training sessions. Accommodation to reversion of the day/night cycle lasted a week.

The experiments were conducted in accordance with the European Communities Council Directive of 24 November 1986 (86/609/EEC) and were reviewed and approved by the Animal Welfare Committee of the Institute of Experimental Medicine.

Rodents were divided in two groups each one containing four members. Group A being subjected to anesthesia while group B was used as control.

Animals in group A recived the following drug mixture: $5 \mathrm{ml}$ of ketamine $+5 \mathrm{ml}$ of xylazine $+2 \mathrm{ml}$ of piplophen $+8 \mathrm{ml}$ of saline. We administered $2 \mathrm{ml} / \mathrm{kg}$ body weight of the mixture intraperitoneally and performed the testing 24 hours after the anesthesia. The depth of anesthesia was monitored by examining the corneal reflex and paw withdrawal reflex. Group B was taken as control. Subjects in both groups underwent a day of partial dietary restrictions, then they were subjected to the experimental phase, during which food restriction was mantained.

There was a training phase and a testing phase. During the training phase we could study the learning ability of the two groups and during the test phase we tested their impulsiveness.

The sessions in the testing phase were carried out in the same period of the day like that ones in the training sessions.

Learning ability study and the impulsivity study was conducted as follows, using the "Delay discounting" apparatus (a device capable of programming time and period of the delaying of food gratification release) [19].

Experiments assessing impulsive behavior were conducted using automated operant chambers, equipped with two nose-poke holes (holes where pellets of food were released, pellets which in this study were named "reward").

The holes were also equipped with infrared sensors and LED lighting, light within the chamber and feeding device with a reservoir containing pellets of food (Med Associates, St. Albans, VT, United States of America). The chambers were placed inside some soundproofed wooden cabins, and were controlled by computers running OS MED-PC IV software (Med Associates, St. Albans, VT, USA) [19].

Rat's answer was considered touching the nose-poke hole. One answer was rewarded with pellets of food of $45 \mathrm{mg}$ each (small reward), while another activating of the nosepoke hole released five pellets of $45 \mathrm{mg}$ each (high reward). Both types of rewards were presented immediately after 
rat's answer and were followed for a period of 25 seconds timeout, during which the light in the operating chamber was switched on. The light in the room was used as a signal that might be associated with reward release after the rat touched one of the nose-poke holes. During the timeout period, the answers were not rewarded but recorded.

To avoid rat's preference response for a certain part of the feeder hole, he was rewarded with five pellets in different holes randomly assigned either to the right or to the left side of the animal. Animals were placed in the same room with the hole assigned to the same side of the nose-poke hole, through the experiment.

We recorded al nose-poke holes activation, like nosepoke holes with small and big reward and also evaluated the number of inappropriate responses (which means actioning the nose-poke holes during the timeout period and the seted periods of delayed released of the pellets).

During the training phase, rats were placed in operant chambers 30 minutes per day, 5 consecutive days. During training/learning phase, the growing percent of preference for greater reward indicates learning (e.g. animals learn to differentiate between hole where if they touch their nose a greater reward will be given and the less reward dispensing).

After two days of rest, animals were subjected to testing phase. During this phase each animal was placed in a room for 30 minutes per day, 8 consecutive days. The procedure was similar to that described in training step, but a delay was introduced before the release of the big reward. The delay of the reward releasing has been established for each daily session and was progressively increased every day as follows: 10, 20 30, 45, 60, 80, 100 and 120 seconds. Responses during these delay periods were not rewarded, but they were recorded by the software.

After each session, the rooms were cleaned with $70 \%$ ethanol and dried with paper towels. All the experiments were performed in the first hours of the light phase.

Data analysis: data where presented as mean \pm standard error of the mean. Behavioral variables of the test with the delay time (preference for greater reward and inappropriate responses) were analyzed using ANOVA analysis of variance (factor 1 was represented by group and repeated factor it was the day or was represented by the delay).

Duncan tests were performed for post-hoc analysis, when a main effect was significant and for multiple comparisons we applied the Bonferroni corrections. We considered statistically significant values of $\mathrm{p}$ less than 0.05 .

\section{Results}

Preference for great reward was manifested more frequent during the 5 days training, suggesting that the animals learned the task (differentiation between large and small rewards). During the training sessions to assess learning ability we recorded preference in nose-poke hole associated with higher reward (greater preference for reward). Greater magnitude increases in preference for higher reward indicates a faster learning process.
As a slight difference in preference for higher reward was noted between the two groups, in the last day of the training phase (see table I, table II and figure 1), three of four anesthetized animals chose large reward versus 2 from control group, preference for higher reward during the test phase was expressed as percentage of the elections for greater reward from the last day of the training phase.

\section{Learning ability study}

Statistical results for the preference for greater reward (associated with learning) for both groups of rats were: medium for anesthetized rats group was 0.893 with a standard error of 0.005 and the average of control group was 0.735 , error 0.142 . The value we obtained for $\mathrm{p}$ was 0.77 , which was not statistically significant (Figure 1).

\section{Impulsivity test}

For the impulsivity test we obtained a $\mathrm{p}$ value equal to 0.277 , which is not statistically significant (Figure 2). The

\begin{tabular}{ccccccc}
\multicolumn{7}{l}{ Table I. Large reward preference } \\
\hline Animal & Group & Day1 & Day2 & Day3 & Day4 & Day5 \\
\hline 1 & anesthesia & 0.727 & 0.666 & 0.744 & 0.857 & 0.893 \\
2 & anesthesia & 0.8 & 0.555 & 0.666 & 0.7 & 0.892 \\
3 & anesthesia & 0 & 0.5 & 0.944 & 0.707 & 0.878 \\
4 & anesthesia & 0.769 & 0.647 & 0.821 & 0.861 & 0.906 \\
5 & control & 0.571 & 0.6 & 0.833 & 0.75 & 0.333 \\
6 & control & 0.4 & 0.419 & 0.690 & 0.765 & 0.888 \\
7 & control & 0.461 & 0.708 & 0.864 & 0.951 & 0.977 \\
8 & control & 0.545 & 0.733 & 0.666 & 0.818 & 0.740 \\
\hline
\end{tabular}

Table II. Data for large reward preference

\begin{tabular}{lccccc}
\hline Group & $\begin{array}{c}\text { Day 1 } \\
(\text { mean } \\
\pm \mathrm{SE})\end{array}$ & $\begin{array}{c}\text { Day 2 } \\
(\text { mean } \\
\pm \mathrm{SE})\end{array}$ & $\begin{array}{c}\text { Day 3 } \\
\text { (mean } \\
\pm \mathrm{SE})\end{array}$ & $\begin{array}{c}\text { Day 4 } \\
(\text { mean } \\
\pm \mathrm{SE})\end{array}$ & $\begin{array}{c}\text { Day 5 } \\
\text { (mean } \\
\pm \mathrm{SE} \text { ) }\end{array}$ \\
\hline Anesthesia & 0.574 & 0.592 & 0.794 & 0.781 & 0.893 \\
& \pm 0.919 & \pm 0.071 & \pm 0.049 & \pm 0.044 & \pm 0.005 \\
Control & 0.494 & 0.615 & 0.763 & 0.821 & 0.735 \\
& \pm 0.039 & \pm 0.071 & \pm 0.049 & \pm 0.045 & \pm 0.142 \\
\hline
\end{tabular}

Large reward preference

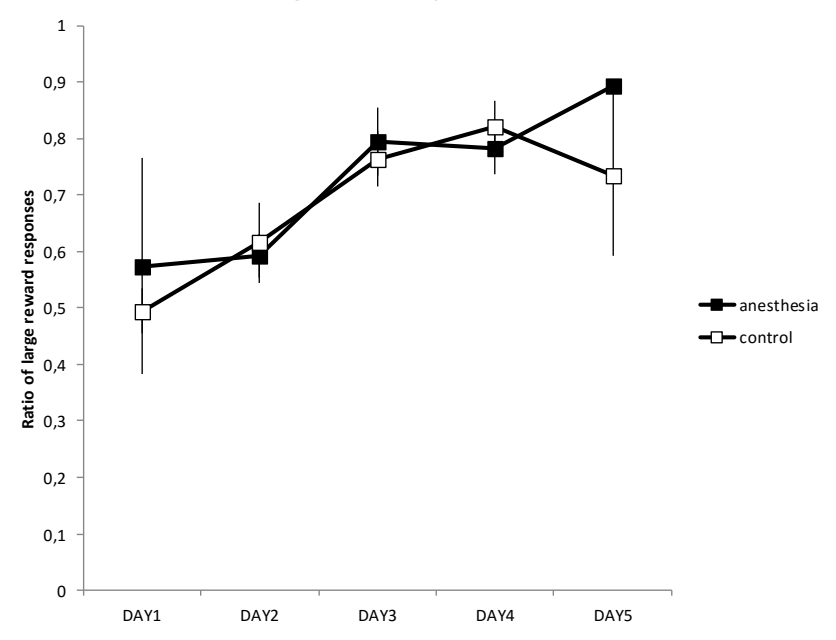

Fig. 1. Study of learning ability-preference for higher reward 


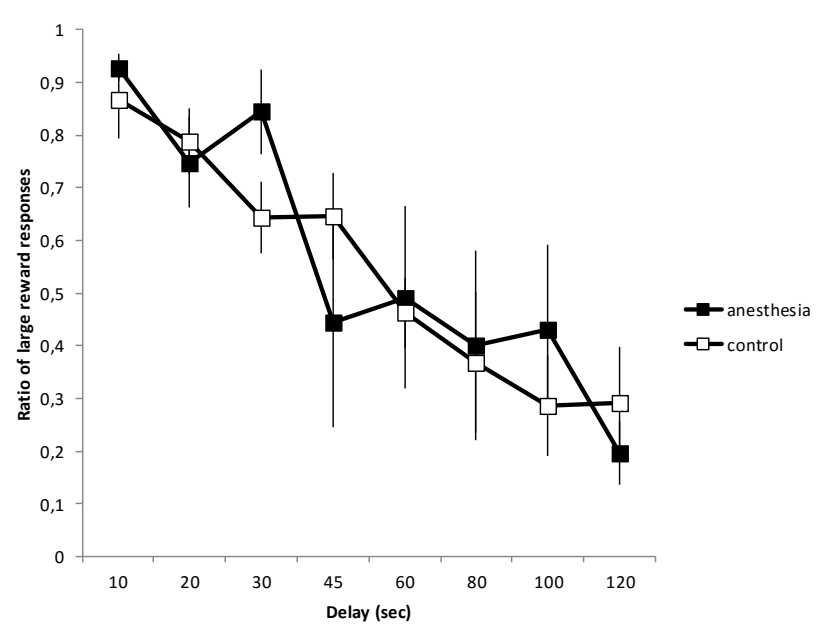

Fig. 2. Study of impulsive behavior-preference for higher reward

results for this test are presented in table III (preference for great reward-anesthetized group) and table IV (preference for great reward-control group).

We also calculated inappropriate responses given by the rats which mean touching the nose-poke holes during the timeout periods and during delay periods and we found the following results, see table $\mathrm{V}$. The results for control group are shown in table VI. The $\mathrm{p}$ value was not statistically significant, it's value being equal to $0.964(>0.05)$ (Figure 3).

We observed a decrease in preference for larger reward in both groups of animals, which indicates an impulsive choice (e.g. inability to wait/choose alternatives or to take in account the future consequences of a response). For this test all animals showed a decrease in preference for higher reward, without any notable effect in anesthetized rats.

Table III. Statistical results for the impulsivity test-preference for great reward-anesthetized group

\begin{tabular}{cc}
\hline Delay (in seconds) & Mean \pm SE \\
\hline 10 & $0.927 \pm 0.026$ \\
20 & $0.747 \pm 0.085$ \\
30 & $0.843 \pm 0.091$ \\
45 & $0.443 \pm 0.197$ \\
60 & $0.491 \pm 0.172$ \\
80 & $0.400 \pm 0.179$ \\
100 & $0.431 \pm 0.160$ \\
120 & $0.196 \pm 0.059$ \\
\hline
\end{tabular}

Table IV. Statistical results for the impulsivity test-preference for great reward-control group

\begin{tabular}{cc}
\hline Delay-in seconds & Mean \pm SE \\
\hline 10 & $0.868 \pm 0.074$ \\
20 & $0.787 \pm 0.063$ \\
30 & $0.643 \pm 0.069$ \\
45 & $0.646 \pm 0.081$ \\
60 & $0.462 \pm 0.066$ \\
80 & $0.367 \pm 0.134$ \\
100 & $0.285 \pm 0.096$ \\
120 & $0.292 \pm 0.105$ \\
\hline
\end{tabular}

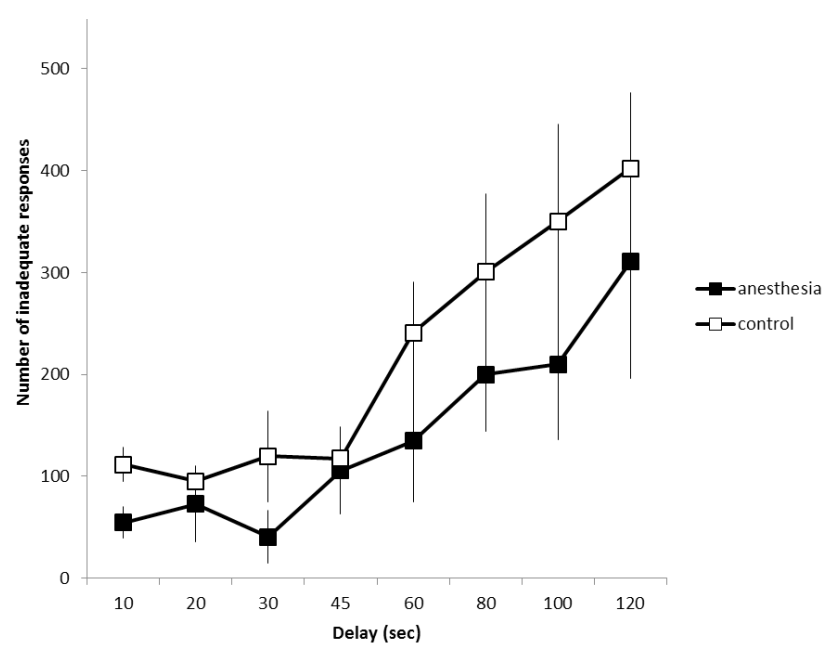

Fig. 3. Study of impulsivity-inappropriate responses

During the test phase the increasing number of inadequate responses (actioning the pellets releasing hole during delays and timeout periods-the 25 seconds period after a response when another answer does not lead to reward) represents motor impulsivity (inability to inhibit a response to a stimulus from the surroundings). During this phase all animals showed an increasing number of inadequate responses over time, but there was no difference between the anesthetized and unanesthetized group, therefore general anesthesia does not increase impulsiveness.

\section{Discussions}

We chosed male adults because there are proofs that feminine sex hormones and their variations such as estrogen and progesterone during the pregnancy period can mediate impulsive and hesitant female subject behavior [20].

Table V. Statistical results for inappropriate responses-anesthetized group

\begin{tabular}{cc}
\hline Delay-in seconds & Mean \pm SE \\
\hline 10 & $54.5 \pm 15.54$ \\
20 & $73 \pm 10.83$ \\
30 & $40.5 \pm 44.70$ \\
45 & $105.5 \pm 30.68$ \\
60 & $134.75 \pm 49.57$ \\
80 & $199.75 \pm 76.84$ \\
100 & $210 \pm 95.61$ \\
120 & $310.5 \pm 74.79$ \\
\hline
\end{tabular}

Table VI. Statistical results for inappropriate responses-control group

\begin{tabular}{cc}
\hline Delay-in seconds & Mean \pm SE \\
\hline 10 seconds & $111.66 \pm 16.85$ \\
20 seconds & $95.33 \pm 10.83$ \\
30 seconds & $119.66 \pm 44.70$ \\
45 seconds & $117.66 \pm 30.68$ \\
60 seconds & $241 \pm 49.57$ \\
80 seconds & $300.66 \pm 76.84$ \\
100 seconds & $350.33 \pm 95.61$ \\
120 seconds & $402 \pm 74.79$ \\
\hline
\end{tabular}


The relatively small number of animals, is mainly due to the fact that inbred strains laboratory animals show a very reduced individual variability, thus they show genetic stability, are isogenic, homozygous with universal distribution so it is possible to compare different studies because rat populations are uniform [21].

This study was conducted in animals due to their lower variability when we compare rats with human subjects who have a lot of incontrollable variables [18].

The light in the room was used as a signal that might be associated with reward release after rat touched one of the nose-poke holes. It is a common practice to associate visual cues and auditory feedback to accelerate learning of operant conditioning procedures. [22]

At the end of training session, animals to responded on the hole associated with higher rewards, fact that is concordant with approximately $90 \%$ of all trials. [23]

During the test phase subjects changed their preference for food from higher reward hole but with later release of the pellets to the hole with the smaller bounty but released on time, which was expected and also observed in previous studies. [24]

During the test phase, the preference for higher reward was indicative for non-impulsive behaviour. This variable is negatively associated with impulsive choice, which refers to an inability to prefer a biger reward which was released later but a smaller, immediately released one. [25]

The number of inappropriate responses reflects the number of premature, impulsive responses. Using this method we were able to measure and asses motor impulsiveness, which is defined as the inability to inhibit inappropriate actions. [25]

The results of our study differ from other trials on animals where rats were repeatedly exposed to anesthesia, when it was noticed that repeated pentobarbital anesthesia in rats (eight fold) affected their behavior towards increased impulsivity, which was assesed by changes in reaction time. This fact was more obvious in the elderly rat population [18]. One explanation of these findings is that in our experiment animals were exposed to anesthesia just once and not repeatedly. Another explanation may be the fact that impulsivity was investigated and expressed different; in number of small reward preferences and inadequate responses in our study design and in changes in reaction time in citated study design.

It seems that pentobarbital anesthesia in rats affects central cholinergic function in hippocampus region. This effect was evaluated (Hanning and Jhonson, 2003) by measuring the isotope $[3 \mathrm{H}]$ epibatidine and [125]alfa-bungarotoxin on brain samples on both anesthetized and unanesthetized rats [26]. The binding of these compunds to nicotinic receptors was also investigated, as well as acethylcoline transferase activity. It was found that after the anesthesia the binding to the nicotinic cholinergic receptor was reduced at the cortical level in the studied area. This fact could have implications in postoperative cognitive functioning [26].
Using an anesthetic combination that includes ketamine we obtained different results than other studies [27] which involved the effect of ketamine anesthesia in rats and where even a subanesthetic dose of ketamine produced more behavioral abnormalities and deficiencies in sensorimotor performance [15]. The link between the increasing cerebral activity induced by low doses of this substance and behavioral changes like cognitive disfunctions and high motor activity was investigated. It resulted that ketamine increased rats' locomotor activity in a dose-dependent manner with a more prolonged effect in higher doses [28].

A dose of $12 \mathrm{mg} / \mathrm{kg}$ of ketamine affected the working memory while a lower dose of $8 \mathrm{mg} / \mathrm{kg}$ had no effect on it. Although low doses of ketamine did not induce memory deficits or hiperlocomotion they affected prepulse stimulation which is regulated by corticolimbic interconnections [28].

The above-mentioned substance interrupts cell activity in cortical areas and hypothalamus and therefore disturbs the flow of information to other interconnectd regions of the limbic system and prefrontal cortex such as nucleus acumbens. This effect leads to behavioral changes when subjects are exposed to higher dose of anesthetic [28]. The resulted differeneces between the above mentioned study and ours may be due to the lower dose of ketamine that we used in combination. If we look closer we see that negative results in Gabor's study are more obvious when a higher dose of ketamine is used.

The negative effects have been observed when rats had to complete a maze test and high doses were used. Shortterm memory and learning ability of spatial navigation tasks were impaired [29].

However these negative effects of ketamine are more common in rats population exposed to ketamine anesthesia in early age, for example in two months of life, when high dose of this substance $(75 \mathrm{mg} / \mathrm{kg})$ has a greater potential to induce apoptosis in hippocampus CA1 region cells and in the girus cinguli cells [30]. As our experiment exposed adult rats to anesthetic substance we noticed no differences in learning ability between anesthetized and control group.

Once again it has to be mentioned that neurocognitive impairment is ketamine dose-dependent. It is the locomotor activity, which is affected while there is no effect on the dark/light cycle. Impairment in work memory is also dosedependent. The expression of $\mathrm{C}$-fos gene in various regions of the limbic system indicates that dose-dependent effects of ketamine may be related to changes in hippocampus and tonsilar region activity [28].

Ketamine-xylazine is also an often-used anesthetic drug combination in rodents. Ordek, Groth and Sahin in their paper "Differential effects of ketamine/xylazine anesthesia on the cerebral and cerebellar cortical activities in the rat" published in 2013, investigated the oxigen partial pressure in the brain of anesthetized and unanesthetized rodents. Using magnetic resonance imaging to measure cerebral blood flow and cerebral oximetry electron paramagnetic 
resonance to study cerebral perfusion in the frontal region of the rats subjected to ketamine-xylazine anesthesia. It was noticed that this combination resulted in decrease with 25 $65 \%$ of cerebral blood flow in a region dependent manner. So there were differences between cerebral regions. As the fact that using ketamine for anesthesia will increase cerebral blood flow and the lowering of cerebral oxygen partial pressure when using the ketamine-xylazine combination is due to xylazine. This changes lead to changes in the hypothalamus and limbic system functioning and also contributes to occurrence of postoperative neurocognitive dysfunction in anesthetized rats $[31,32]$.

Xylazine and ketamine does not act only at the cortical and limbic structures but their combination also suppresses evoked potentials in the cerebellum. Although the affected components at this level are those with frequencies above $30 \mathrm{~Hz}$ [33].

In the awake, quiet animal potential activity in the brain takes place on a range of up to $800 \mathrm{~Hz}$ at the level of cerebellum and up to $200 \mathrm{~Hz}$ in the motor cortex level. The ketamine-xylazine combination suppresses the majority of the activity of the interneuronal circuits at the cerebellar cortex, not in the motor cerebral cortex [34].

When comparing our study results with others, we can say that they differ from those of citated ones. This may be due to that above citated studies have used repeated or greater doses of anesthetic substances.

\section{Limitation of this study}

Although the present study has brought some preliminary findings, the main flaw of it's design is the small number of animal subjects. There remained much to be done, still our research generated an interesting finding in what concerns general anesthesia's influence on rodent behaviour in the field of impulsivity and neurocognitive processes.

\section{Conclusions}

Anesthesia had no effect on learning ability.

During the test phase all animals showed an increasing number of inadequate responses over time, but there was no difference between the anesthetized and unanesthetized group. The conclusion of the study was that general anesthesia does not increase impulsiveness.

\section{Conflicts of interest}

The authors report no conflicts of interest.

\section{References}

1. Müller SV, Krause N, Schmidt M, Münte TF, Münte S. Cognitive dysfunction after abdominal surgery in eldery patients. Z Gerontol Geriatr. 2004;37(6):475-485.

2. Bedford PD. Adverse cerebral effects of anesthesia on old people. Lancet. 1955;269(6884):259-263.

3. Rundshagen I. Postoperative cognitive dysfunction. Dtsch Arztebl Int. 2014;111(8):119-125.

4. Moller JT, Cluitmans P, Rasmussen LS, et al. Long-term postoperative cognitive dysfunction in the eldery: ISPOCD1 study. Lancet. 1998;351(9106):857-861.
5. Rasmussen L, Johnson T, Kuipers $H$, et al. Does anesthesia cause postoperative cognitive dysfunction? A randomised study of regional vrsus general anesthesia in 438 eldery patients. Acta Anaesthesiol Scand. 2003;47(3):260-266.

6. Koichi Y, Dima G. Postoperative maladaptive behavioural changes in children.Middle East J Anaesthesiol. 2011;21(2):183-189.

7. Kain Z, Wang S, Mayes L, Caramico L, Hofstadter M. Distress during the induction of anesthesia and postoperative behavioural outcomes. Anesth Analg. 1999;88(5):1042-1047.

8. Kain Z, Caldwell A, Maranets I, et al. Preoperative anxiety and emergence delirium and postoperative maladaptative behaviors. Anesth Analg. 2004;99(6):1648-1654.

9. Winstanley CA, Theobald DE, Cardinal RN, Robbins TW. Contrasting roles of basolateral amygdala and orbitofrontal cortex in impulsive choice. J Neurosci. 2004;24(20):4718-22.

10. Reynolds B, Richards JB, Dassinger M, de Wit H. Therapeutic doses of diazepam do not alter impulsive behaviour in humans. Pharmacol Biochem Behav. 2004;79(1):17-24.

11. Stratmann G, Sall J, May L, Loepke A, Lee M. Beyond anesthetic proprieties: the effects of isoflurane on brain cell death, neurogenesis, and long-term neurocognitive function. Anesth Analg. 2010;110(2):431437.

12. Huang $H$, Liu $C$, Sun $J$, et al. Ketamine affects the neurogenesis of the hippocampal dentate gyrus in 7-day-old rats. Neurotox Res. 2016;30(2):185-198. doi: 10.1007/s12640-016-9615-7.

13. Hehar H, Yeates k, Kolb B, Esser MJ, Mychasiuk R. Impulsivity and concussion in juvenile rats: examining molecular and structural aspects of the frontostriatal pathway. PLoS One. 2015;10(10):e0139842. doi: 10.1371/journal.pone.0139842.

14. Hagberg $H$, Peebles D, Mallard C. Models of white matter injury: comparison of infectious, hypoxic-ischemic, and excitotoxic insults. Ment Retard Dev Disabil Res Rev. 2002;8(1):30-38.

15. Loepke AW, Soriano SG. An assessment of the effects of general anesthetics on developing brain structure and neurocognitive function. Anesth Analg. 2008;106(6):1681-1707. doi: 10.1213/ ane.0b013e318167ad77.

17. Culley D, Baxter M, Yukhananov R, Crosby G. Long-term impairment of acquisition of a spatial memory task following isoflurane-nitrous oxide anesthesia in rats. Anesthesiology. 2004;100(2):309-314.

16. Loepke A, McGowan F, Soriano S. CON: The toxic effects of anesthetics in the developing brain: the clinical perspective. Anesth Analg. 2008;106(6):1664-1669. doi: 10.1213/ane.0b013e3181733ef8.

18. Zheng W, Ycu E. A fully automated and highly versatile system for testing multi-cognitive functions and recording neuronal activities in rodents. J Vis Exp. 2012;(63):e3685. doi: 10.3791/3685.

19. Cheryl A, Llanezza D. Progestogens and estrogen influence impulsive burying and avoidant freezing behavior of natural Fryealy cycling and ovariectomized rats. Pharmacol Biochem Behav. 2009;93(3):337-342.

20. Festing $M$-Inbred strains, in Baker $H$, Russell J, Weisbroth $H$. The Laboratory Rat, Volume I: Biology and Disease. Academic press. 1979. pg. 56.

21. Bokland A, Honig W, Jolles J. Long-term consequences of repeated pentobarbital anaesthesia on choice reaction time performance in ageing rats. Br J Anaesth. 2001;87(5):781-783. doi: 10.1093/bja/87.5.781

22. Panlilio LV, Ferré S, Yasar $S$ et al. Combined effects of THC and caffeine on working memory in rats. Br J Pharmacol. 2012;165(8):2529-2538.

23. Adriani W, Caprioli A, Granstrem O, Carli M, Laviola G. The spontaneously hypertensive-rat as an animal model of ADHD: evidence for impulsive and non-impulsive subpopulations. Neurosci Biobehav Rev. 2003;27(7):639651.

24. Adriani W1, Seta DD, Dessì-Fulgheri F, Farabollini F, Laviola G. Altered profiles of spontaneous novelty seeking, impulsive behavior, and response to $\mathrm{D}$-amphetamine in rats perinatally exposed to bisphenol $\mathrm{A}$. Environ Health Perspect. 2003;111(4):395-401.

25. Kim S, Lee D. Prefrontal cortex and impulsive decision making. Biol Psychiatry. 2011;69(12):1140-1146.

26. Hanning C, Blokland A, Jhonson M, Perry E. Effects of repeated anesthesia on central cholinergic function in the rat cerebral cortex. Eur $J$ Anesthesiol. 2003;20(2):93-97.

27. Gabor I, Fokkema DS, Den Boer JA, Ter Horst GJ. Dose-response characteristics of ketamine effect on locomotion, cognitive function and central neuronal activity. Brain Research Bulletin. 2006;69:338-345.

28. Gabor I, Dirk S, Johan A, Gert J. Dose-response characteristics of ketamine effect on locomotion, cognitive function and central neuronal activity. Brain Res Bull. 2006;69(3):338-345.

29. Zhu X, Hao X, Luo J, et al. Propofol inhibits inflammatory cytokine- 
mediated glutamate uptake dysfunction to alleviate learning/memory impairment in depressed rats undergoing electroconvulsive shock. Brain Res. 2015;1595:101-109. doi: 10.1016/j.brainres.2014.07.046

30. Ordek G, Groth JD, Sahin M. Effect of anesthesia on spontaneous activity and evoked potentials of the cerebellar cortex. Conf Proc IEEE Eng Med Biol Soc. 2012;2012:835-838. doi: 10.1109/EMBC.2012.6346061.

31. Ordek G, Groth J, Sahin M. Differential effects of ketamine/xylazine anesthesia on the cerebral and cerebellar cortical activities in the rat. $J$ Neurophysiol. 2013;109(5):1435-1443. doi: 10.1152/jn.00455.2012.
32. Bengtsson F, Jörntell $H$. Ketamine and xylazine depress sensory-evoked parallel fiber and climbing fiber responses. J Neurophysiol. 2007;98:16971705.

33. Krysl D, Deykun K, Lambert L, Pokorny J, Mares J. Perifocal and remote blood-brain barrier disruption in cortical photothrombotic ischemic lesion and its modulation by the choice of anesthesia. J Physiol Pharmacol. 2012;63(2):127-132.

34. Alessandri $B$, Bättig K, Welzl H. Effects of ketamine on tunnel maze and water maze performance in the rat. Behav Neural Biol. 1989;52(2):194212. 